\title{
ENSAYO
}

ESSAY

\section{Una reflexión sobre urbanismo táctico, periferia marginal y participación ciudadana}

\author{
A reflection on tactical urbanism, marginal periphery \\ and citizen participation
}

John Braulio Contreras-Cerón* (1) https://orcid.org/0000-0003-2265-6767

Citar este artículo como: John Braulio Contreras-Cerón, J. B. (2021). Una reflexión sobre urbanismo táctico, periferia marginal y participación ciudadana. Revista Nodo, 15(30), pp. 74-88.

\section{Resumen}

Este escrito presenta algunos elementos teóricos que hacen parte del marco conceptual del proyecto de investigación "Intervenciones tácticas en periferias marginales y la apropiación ciudadana del sector el Polvorín de San Juan de Pasto, Colombia"; su objetivo fue determinar los impactos e incidencia de estas categorías en realidades locales, en las formas de insertar iniciativas y metodologías de vanguardia que se relacionan con lo físico espacial y sus impactos inmediatistas, en la búsqueda de desarrollos urbanos como referencia estética, que puedan convertirse en otra forma de pensar las problemáticas de los asentamientos periféricos. El proyecto en mención fue una iniciativa de investigación entre la Universidad Católica de Pereira y la Universidad Centro de Estudios Superiores María Goretti (CESMAG), que en principio buscaba desarrollar una propuesta de intervención físico espacial y socio-cultural desde lo participativo en los barrios El Rocío Alto y Caracol la Curva, de Pereira y El Polvorín, de Pasto (Colombia). Sin embargo, de momento, sólo se ha podido llevar a cabo la experiencia en este último sector. Teniendo en cuenta lo anterior el propósito de este ensayo es presentar una reflexión teórica sobre el urbanismo táctico y la participación ciudadana en entornos marginales en donde cobran importancia aspectos como el canje de uso, las memorias cotidianas, la valoración de los intersticios subutilizados y la búsqueda de una apropiación por parte de la comunidad en el restablecimiento del paisaje urbano existente.

Palabras clave: Diseño urbano, Participación comunitaria, Zona Urbana, Planificación urbana.

\section{Abstract}

This paper presents some theoretical elements that are part of the conceptual framework of the research project "Tactical interventions in marginal peripheries and citizen appropriation of the

Fecha de recepción: 28 de marzo de 2020 • Fecha de revisión: 10 de septiembre de 2020

Fecha de aceptación: 18 de octubre de 2020 - Fecha de publicación: 9 de abril de 2021

* Arquitecto y Planificador Urbano Regional. Candidato a Magíster en Planificación Territorial y Gestión Ambiental en la Universidad de Barcelona, España. Profesor de tiempo completo del Programa de Arquitectura, Facultad de Arquitectura y Bellas Artes, Universidad Centro de Estudios Superiores María Goretti (CESMAG). Correo electrónico: email: jbcontreras@ unicesmag.edu.co 
Polvorín de San Juan de Pasto sector, Colombia"; whose objective was to determine the impacts and incidents of these categories in local realities, in the ways of inserting cutting-edge initiatives and methodologies that are also related to the physical and spatial aspects and their immediate impacts in the search for urban developments as an aesthetic reference, which can become another way of rethinking the problems of peripheral settlements. The project in question started from an research initiative between the Catholic University of Pereira and the María Goretti University Center for Higher Studies (CESMAG) that initially sought to develop a proposal for physical, spatial and socio-cultural intervention from the participatory perspective in the neighborhoods El Rocío Alto and Caracol la Curva, from Pereira and El Polvorín, from Pasto (Colombia). However, so far only the experience in the latter sector has been carried out. Taking into account the above, the purpose of this essay is to present a theoretical reflection on tactical urbanism and citizen participation in marginal environments where aspects such as exchange of use, daily memories, the valuation of underused interstices and the search become important of an appropriation by the community in the restoration of the existing urban landscape.

Keywords: Urban design, Community participation, Urban Zone, Urban decentralization, Urban planification.

\section{Introducción}

Las intervenciones tácticas, los proyectos pilotos de impacto o el urbanismo táctico se han convertido en una tendencia global que busca cambios intempestivos y temporales en la cotidianidad de espacios urbanos problemáticos. Su finalidad es motivar la reflexión con respecto al canje de uso o morfología propuesta con su correspondiente aceptación, participación y apropiación por parte de la población con el ánimo de contribuir al mejoramiento del paisaje urbano impactado. Y esa fue una de las intenciones que se tuvo al momento de formular la propuesta "Intervenciones tácticas en periferias marginales y la apropiación ciudadana del sector el Polvorín San Juan de Pasto" ${ }^{1}$ cuya base es el desarrollo de un taller de diseño con estudiantes del Programa de Arquitectura de la Universidad Centro de Estudios Superiores María Goretti (CESMAG), en que en el abordaje del contenido temático la Vivienda social se intervienen sectores marginales que se convierten en el escenario ideal para llevar a cabo esta indagación. Todo ello en la búsqueda de un diálogo de saberes entre la comunidad beneficiaria y la academia, que al final deberá evidenciar elementos del urbanismo táctico en consonancia con procesos de participación comunitaria local.

$\mathrm{Al}$ momento de hablar de intervenciones tácticas es necesario hacer una revisión de algunos conceptos y teorías relacionadas con el urbanismo táctico, el Design thinking (Pensamiento de Diseño), el Hacking the city (Hackeando la ciudad), el diseño participativo y la participación ciudadana para identificar aquellas estrategias que puedan ser aplicadas a una realidad específica. Más allá, la revisión de experiencias de participación ciudadana, de intervenciones piloto en el territorio, de diseño participativo, etc., pueden servir también de referentes estéticos para formular propuestas cercanas y sensibles que permitan la cualificación del paisaje y la vida urbana; tal como enuncian Brenna et al. (2020) al presentar los resultados de un amplio análisis de la literatura en donde resaltan las medidas, en términos de soluciones tecnológicas y técnicas de gestión, que son investigadas e implementadas en la actualidad.

Teniendo en cuenta lo dicho hasta aquí las motivaciones académicas de este artículo se enfocan principalmente en identificar criterios de algunos autores que han reflexionado sobre los temas

1 En principio este proyecto también incluía los barrios El Rocío Alto y Caracol la Curva, de Pereira, pero por motivos logísticos no se ha podido realizar aún dicha experiencia. Por ello sólo se está trabajando con el sector El Polvorín, de la ciudad de Pasto. 
mencionados, de tal suerte que sea posible aproximarse a interrogantes como ¿Cuáles son las metodologías de urbanismo táctico actualmente utilizadas? ¿Cuáles son las relaciones entre el urbanismo táctico y participación ciudadana? ¿Por qué utilizar el urbanismo táctico en una periferia marginal? ¿Cómo beneficiaría a la comunidad el urbanismo táctico?

\section{Algunos elementos metodológicos}

En la realización de esta revisión teórica se utilizó un protocolo para la búsqueda de referencias en bases de datos de plataformas científicas. Scopus constituyó el inicio de la cadena de búsqueda con 3.335 artículos encontrados que posteriormente, aplicando criterios de inclusión (IC) y exclusión (EC) tales como artículos de acceso libre (Open Acces), escritos durante los últimos cuatro años (IC1) y limitando a las artes y humanidades (EC3) y en idioma español (EC2), arrojó un resultado de 31 artículos. Science direct fue otra plataforma científica de relevancia en la que se aplicó criterios de inclusión (IC) y exclusión (EC) similares a los señalados arriba y arrojó un resultado de 11 artí- culos. Y, por último, también se acudió al gestor bibliográfico Mendeley que dio un resultado total de 9 libros. Posterior a esto se hizo un ejercicio de exclusión (EC), (EC3), (EC2) y se llegó a 28 contenidos que se relacionan directamente con los intereses temáticos de este trabajo, tal como se muestra en el Diagrama 1.

\section{De la marginalidad, la participación ciudadana y el urbanismo táctico}

Es de especial interés que la arquitectura se enfoque en aspectos relacionados con el territorio y sus fenomenologías; por eso es importante que se traten aspectos sobre los paradigmas existentes desde la precariedad en las intervenciones urbanas en periferias marginales. En este sentido, las intervenciones en espacios periféricos son entornos ideales para que lo físico espacial sea representado en el desarrollo del paisaje y que al final pueda ser apropiado por actores sociales y que, permeado con procesos de participación y apropiación comunitaria, desemboque en cierta garantía de avances que mejoren la vida urbana. Es relevante anotar, sin embargo, que los cambios

Diagrama 1. Proceso de búsqueda de documentos

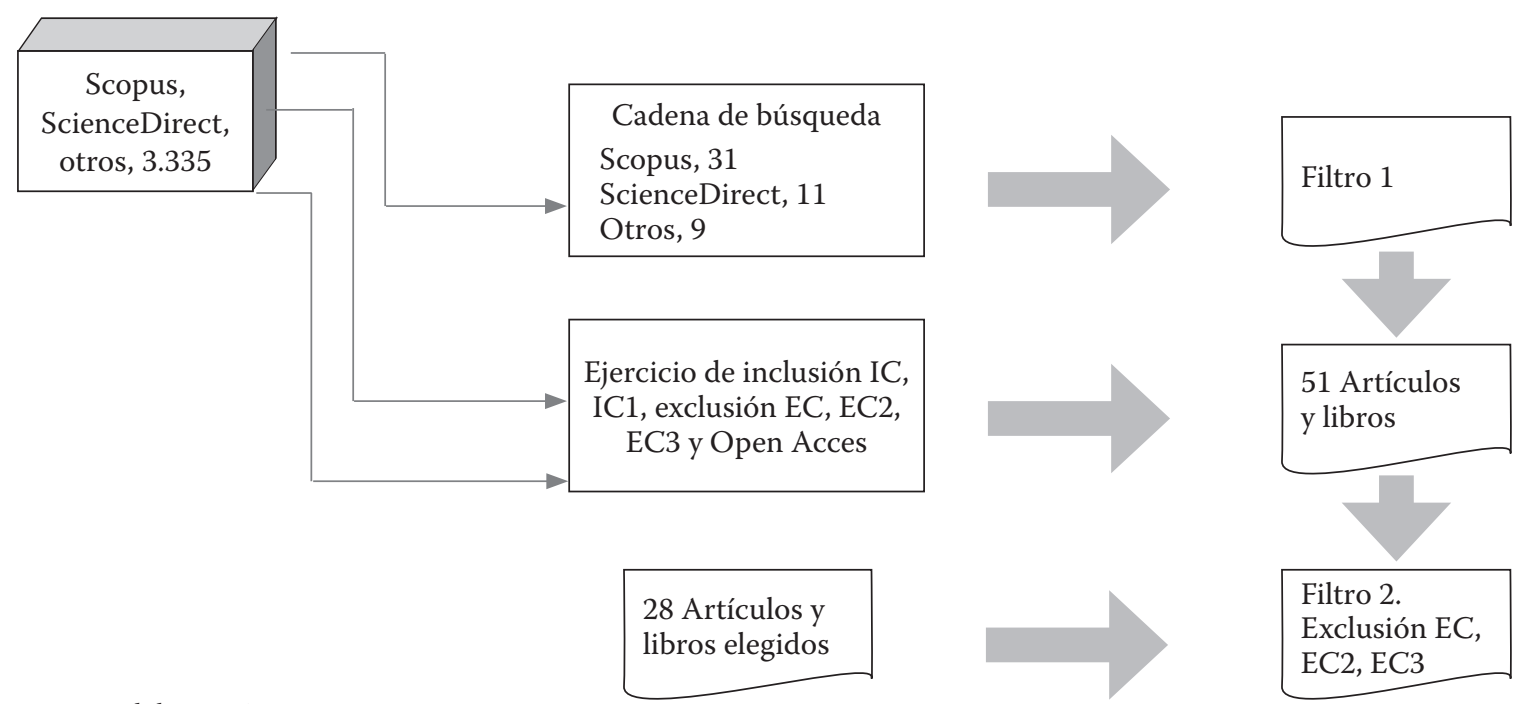

Fuente: elaboración propia. 
espaciales pueden obedecer a mutaciones locales resultado de lógicas sociales propias, que a veces son imperceptibles o pasan desapercibidas en la cotidianidad de los habitantes de un sector.

De todas maneras es necesario tener en cuenta que lo físico espacial hace parte de las especificidades y fenomenologías de la ciudad; por lo tanto lo marginal, lo periférico, los asentamientos humanos de crecimiento espontáneo son una constante que no puede dejarse al margen si se plantean posibles desarrollos desde lo local y desde lo académico, que busquen impactar positivamente en comunidades alejadas de las centralidades y que tienen las problemáticas típicas de la ciudad intermedia latinoamericana.

\section{Sobre la marginalidad urbana}

La marginalidad urbana se podría describir de forma general como periferias o guetos con aislamiento o segregación socio espacial, con problemáticas de tenencia de tierras, con déficit de infraestructuras; con bajo poder adquisitivo, desempleo e informalidad de sus habitantes; con problemas de cohesión social que se ve como el resultado de lógicas y estéticas populares, que redundan en un paisaje urbano no necesariamente incluido en la regularización normativa; con vulnerabilidad latente a lo cual se suma el complemento perverso de una visión con prejuicio de ser sectores inseguros, tal como exponen Samper, Ortiz y Soto (2014):

(...) también se cree que la informalidad es equivalente a la pobreza, es un caos espontáneo y que es exclusivo al sur global siendo una manifestación del desarrollo, muy asociado a estos mitos acerca de la informalidad se encuentran los conceptos de carencias, marginalidad y conflicto. En la práctica, la informalidad es el modo prevalente de la urbanización, que funciona en un mercado no regulado y que surge como producto de la acción o inacción del Estado (p. 2).

Así pues, se observan similitudes y elementos comunes que desembocan en términos tales como marginalidad, informalidad, asentamientos subnormales, territorios vulnerables, tugurios, "lugares inseguros", dentro de los imaginarios colectivos de quienes habitan cualquier ciudad. Por ello es necesario impulsar iniciativas que se encuentren en consonancia con "repensar la informalidad" y precisar la marginalidad desde el urbanismo, como el resultado de un asentamiento subnormal de crecimiento espontáneo, que obedece a consecuencias macroeconómicas nacionales de un modelo capitalista y a conflictos internos que empujan a amplios sectores de la población hacia las ciudades, que se convierten en un imán de posibles "progresos personales y económicos". Desde lo académico estos lugares pueden constituir un laboratorio ideal para la indagación sobre el impacto de lo físico espacial y la participación comunitaria en el surgimiento de propuestas de desarrollos locales.

\section{Participación ciudadana}

Los procesos de participación ciudadana en los sectores marginales deben impactar positivamente en las iniciativas, los sueños y anhelos comunitarios; y aunque hoy en día se han convertido en un cliché de la planificación urbana, y se lo confunde con "socialización de proyectos", por dispendiosos y desgastantes que puedan ser para las administraciones o los consultores urbanos, deben apoyarse en éstos si se quieren ver verdaderas apropiaciones ciudadanas en proyectos urbanos.

En el contexto latinoamericano se han llevado a cabo procesos de participación ciudadana que, aunque con falencias, tienen mucho potencial para ser tenidas en cuenta. Sin embargo, como mencionan Noriega, Aburto y Montecinos (2016) en la práctica el Presupuesto Participativo (PP) todavía "no ha logrado incluir del todo a la ciudadanía, observándose en los hallazgos que son las personas jóvenes, sin poder organizativo, las menos favorecidas en términos de participación (p. 203). 
Sea como fuere, merece la pena resaltar la importancia de la participación comunitaria para llevar a cabo iniciativas que contribuyan a la cohesión social y a la inclusión de sectores populares. Todo ello con el ánimo de que se conviertan en potenciales nodos de micro desarrollos de autogestión y apropiación de procesos sociales, que pueden ser evidenciados en lo físico espacial pues, a la larga, también se busca "la reafirmación de las nuevas centralidades" (Signorelli, 2016, p. 103) y una transformación de los paisajes urbanos propuestos desde lo arquitectónico.

Aunque no se enfoque desde la participación ciudadana es importante destacar que, en la mayoría de los asentamientos humanos marginales, existe un alto índice de desplazados que vienen de zonas rurales con sus propios usos y costumbres que pueden ser articulados a los modos urbanos de ciudadanía y que tienen el potencial de aportar a la generación de tejido humano desde la interculturalidad. Desde ese punto de vista, como lo sugiere Ramos-Vidal (2017), el solo hecho de realizar un proceso de visibilizar la comunidad desplazada es una propuesta de inclusión en lo participativo:

(...) en el sentido de realizar un estudio exploratorio transversal con personas desplazadas $(n=30)$ y no desplazadas $(n=32)$ en dos distritos de bajos ingresos de Barranquilla (Colombia). Existe una retroalimentación positiva entre los procesos evaluados, aunque no se observan diferencias significativas entre los grupos desplazados y no desplazados. La dimensión de pertenencia (sentido de comunidad) es la que mejor explica la variación del empoderamiento y la participación en ambos grupos (...) (p. 49).

Si queremos responder preguntas como ¿cuáles son los problemas físico espaciales de la periferia urbana marginal? y ¿cuáles son las oportunidades de mejora en la búsqueda de esfuerzos comunitarios para su desarrollo urbano?, se debe empezar por decir que todo lo relacionado con la participación ciudadana se contempla como autogestión local, procesos de apropiación, interés por mejorar lo comunitario, anhelos comunes de un conjunto de vecinos (Dalla-Torre, 2017). Cuando los desarrollos territoriales locales son incipientes y se cree que la idea de la participación ciudadana es parte de una terminología tecnócrata -aunque sus premisas busquen una democratización del empoderamiento local y su divulgación no se baje de las esferas académica - se hace necesaria una transferencia de conocimiento enfocada al ciudadano inmerso en la problemática local.

En algunos contextos latinoamericanos, los gobiernos locales no pueden intervenir con recursos económicos en asentamientos humanos "ilegales", por eso es necesario los movimientos comunitarios de autogestión que puedan constituirse en fuerzas sociales fuertes en la consecución de mejoras en distintos ámbitos vitales. En ese sentido merece la pena tener en cuenta algunos planteamientos de Cubero (2019) cuando afirma que:

(...) en el contexto de un mundo dominado por la racionalidad económica, nuestra región ha visto surgir los llamados "nuevos" movimientos sociales, que unen fuerzas con antiguas luchas por la tierra e intentan combatir la destrucción y despojo de la naturaleza y la degradación del medio ambiente, al tiempo que defienden la calidad de vida de la población ( p. 135).

Lo anterior también se evidencia en lo enunciado por Pindado (2004) cuando habla de la "posibilidad de que cualquier persona pueda participar en los asuntos públicos de la comunidad de la que forma parte" (p. 135); por tanto, es necesario desplegar medios técnicos, económicos y el instrumental adecuado para que la participación se pueda realizar y sea efectiva. En este sentido si pensamos que la participación ciudadana puede ser efectiva en un asentamiento humano, ese asunto público le compete a toda la comunidad y es susceptible de intervenciones desde varios aspectos; pero en su especificidad no es más que la búsqueda de la mejora de la calidad de vida. Pindado (2004) también propone "analizar la importancia y el rol de la participación ciudadana en las políticas 
públicas ambientales, desde una perspectiva latinoamericana y bajo la categoría central de la conflictividad social" (p. 135).

No importa si las iniciativas de participación son gubernamentales, de las ONG, o de la academia, sino que éstas permitan la generación de nuevas propuestas de políticas públicas para crear espacios de intervención y autogestión local de desarrollo que deben ser tenidos en cuenta; no solo con el propósito de romper paradigmas existentes de visiones gubernamentales paquidérmicas o burocratizadas, sino también para asegurar la continuidad de procesos de participación de todos los actores sociales en diferentes fenomenologías urbanas periféricas. Lo anterior teniendo en cuenta que algunos recorridos burocráticos superan en el tiempo los períodos de gobierno cuatrienales como sucede en Colombia y ello puede originar que se corten procesos participativos.

Vale la pena resaltar que, actualmente, en los procesos de participación ciudadana se están teniendo en cuenta aspectos como la perspectiva de género. En este sentido es de destacar el trabajo de las mujeres como agentes principales que pueden incidir en los cambios y desarrollos urbanos. Por ello también se están llevando a cabo procesos de formación en los diversos ámbitos de la vida citadina en los cuales se introduce la perspectiva de género, tal como advierte Ciocoletto (2014):

(...) se han realizado cursos y talleres de formación, además de asesoría en urbanismo con perspectiva de género y se han elaborado algunos de los proyectos con criterios de género. Existen canales de participación vinculados tanto a la elaboración de programas sociales como a la mejora de algunos espacios del barrio. Se han llevado a cabo especialmente trabajos con mujeres para identificar las necesidades cotidianas. Se ha instalado un espacio físico de referencia en el barrio para interactuar con las personas vecinas, con una programación de actividades que incluye la perspectiva de género (p. 90).
Los procesos de participación ciudadana no solo deben ser teocracia de los textos académicos, sino que deberían existir políticas con procesos más expeditos de participación de tal manera que permita a la gente empoderarse de sus propios desarrollos y que, de verdad, se vuelvan tangibles con indicadores de participación y posibles seguimientos que hagan posible su actualización y corrección a lo largo del tiempo. Y todo ello porque, como enuncia Ziccardi (2004), la intención que subyace en los procesos de "participación ciudadana y en las políticas sociales del ámbito local” es

abrir un diálogo franco, respetuoso y productivo entre los académicos y quienes desde la gestión pública piensan que se pueden obtener mejores resultados si se incluye a la ciudadanía en los procesos de toma de decisiones públicas, a la vez que se puede avanzar en la construcción de una cultura democrática (p. 441).

\section{Urbanismo táctico}

Las intervenciones físico espaciales relacionadas con el urbanismo táctico y que son presentadas como proyectos piloto de bajo costo, que cambian cotidianidades existentes para solucionar una problemática específica urbana, pueden desembocar en procesos de infraestructura de mayor envergadura, con grandes presupuestos y con una apropiación definitiva por parte de la ciudadanía; lo anterior está en consonancia con lo planteado por Gehl (2014), al enunciar que:

(...) pueden generar un genuino estímulo para que la gente camine $o$ ande en bicicleta dentro de la ciudad, y si las personas realmente quieren permanecer dentro del entorno urbano, crear un microclima a la altura de la vista es una tarea fundamental. Hay mucho que se puede hacer. No necesita grandes inversiones, sino más bien algunos requerimientos precisos y de mucha consideración (p. 45).

Esta idea es pertinente cuando las intervenciones tácticas en los territorios buscan impactar y/o 
cambiar positivamente el paisaje urbano existente, en las formas de uso del espacio, las actividades cotidianas, los intersticios urbanos subutilizados; a transformar las estéticas populares que también equivale a romper paradigmas locales periféricos con una mínima instalación urbano arquitectónica, de bajo costo, que genere cierto cambio y a veces incomode la rutina diaria; es, en sí, romper preexistencias espaciales en pos de una mejora de entornos problemáticos. A veces esos procesos de intervención son más largos y trascendentales como, por ejemplo, la peatonalización de Times Square en Nueva York, (Figuras 1 y 2) que cambió radical y positivamente su paisaje urbano (Gené y Ferré, 2014; Stang, 2014).



Figuras 1 y 2. Times Square antes de la intervención urbanística y después de la misma. Fuente: Randomwinner, Pixabay.
Muchas veces estas iniciativas de intervenciones son itinerantes o temporales de bajo costo, con tecnologías mínimas y materiales locales y se realizan sobre espacios e intersticios urbanos subutilizados; esto lleva a una reflexión que puede generar pensamientos críticos sobre la cotidianidad del uso inadecuado o buscar el cambio de funcionalidad espacial que beneficie al peatón desprevenido. En su conjunto implica una mejora y un mayor disfrute de estos entornos que a la larga no es más que el progreso en la calidad de vida local. A veces con el simple hecho de que los vecinos de una calle se pongan de acuerdo para aportar diferentes tipos de lámparas para iluminar una vía oscura, se logran cambios urbanos preponderantes, tal como sucedió en Atenas, Grecia (Figura 3).

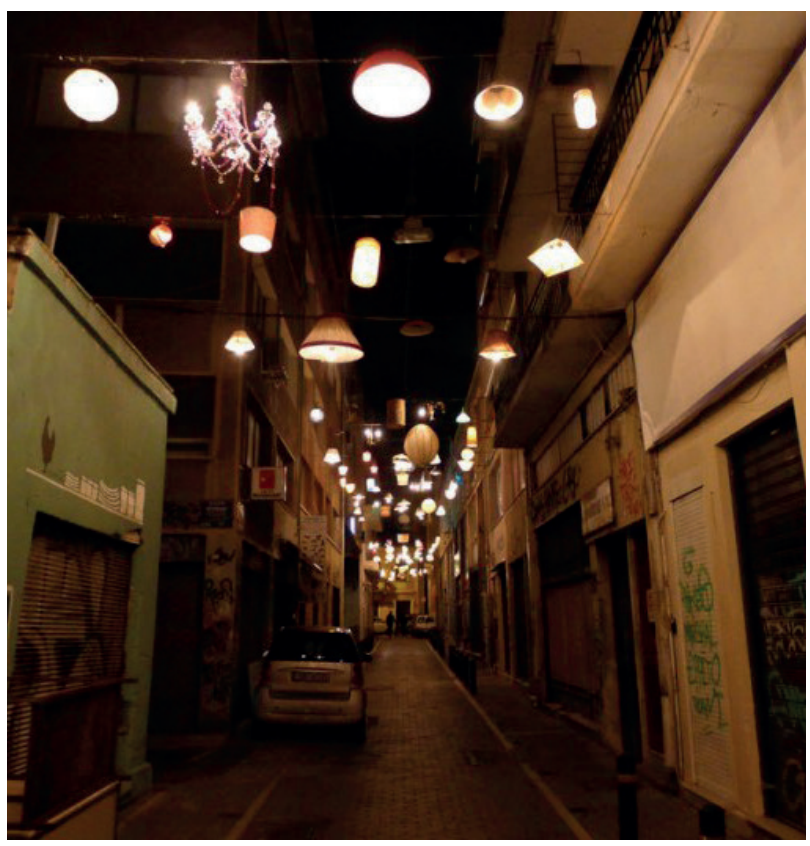

Figura 3. Lámparas donadas por vecinos iluminan una calle de Atenas, Grecia. Fuente: Plataforma Urbana (2013).

Lo dicho hasta aquí se relaciona con lo tratado por Christof (2017) en su texto "El profesional ciudadano, la mediatización y la creación de un dominio público", basado en entrevistas piloto realizadas en Rotterdam (NAC, Reading Room West) y Viena (Paradocks), en donde afirma entre otras cosas, que "para exponer los proyectos como espacios 
vividos entre entornos mediatizados y físicos" (...) es necesario investigar "los valores subyacentes de las intervenciones espaciales, así como la forma en que los organismos gubernamentales se relacionan con sus prácticas" (p. 279). Visto a través de la libertad de acción, el artículo mencionado proporciona información sobre cómo los profesionales y los ciudadanos emplean sus imaginarios sociales y movilizan sus actividades en torno a una agenda para la creación de un dominio público. Aunque no se habla de proyectos piloto de intervenciones -que es el componente fundamental del urbanismo táctico-, sí lo hace de la apropiación de las intervenciones físico-espaciales, aunque no aclara si la premisa de la propuesta es la valoración de proyectos urbanos y el quehacer gubernamental.

En este sentido, Diz (2018) enuncia que para el activismo contemporáneo el cuerpo es el primer territorio para la resistencia en el que

en un contexto internacional de crisis política y socioeconómica, la ciudad se convierte no sólo en el escenario de la acción, sino que también está en juego. El desafío es comprender la resistencia del activismo basada en las interrelaciones del cuerpo y la ciudad (p. 127).

Desde ese punto de vista, el urbanismo táctico se justifica teniendo en cuenta que los escenarios marginales hacen parte de la urbe $y$, por lo tanto, estos sectores no pueden dejarse de lado como paisaje de intervención. A manera de ejemplo merece la pena comentar que, en la ciudad de San Juan de Pasto, departamento de Nariño, Colombia, se intentó llevar a cabo un ejercicio de intervención táctica sobre el parque de Nariño en la margen de la carrera 24 en el que se pretendió, por medio de unos maceteros con plantas ornamentales, recuperar un espacio utilizado tradicionalmente por vehículos para que fuera usado por transeúntes y así cambiar su uso cotidiano; sin embargo, este ejercicio no fue entendido o apropiado por la ciudadanía. Pese a ello es importante valorar esta iniciativa gubernamental que quizá por falta de planeación, elaboración y/o por ausencia de elementos pictóricos sobre la superficie de la calzada, no llamó la atención de los usuarios diarios e impidió transmitir mejor la idea de una nueva espacialidad pública; lo anterior hizo que la ciudadanía no apropiara ni hiciera suyo el proyecto, que debe ser el fin último de una intervención táctica.

\section{Design Thinking (Pensamiento de Diseño)}

Para comprender este concepto vale la pena visitar la página $w^{2} b^{2}$ en donde se muestran todos sus procesos; es muy completa y muy práctica para quien quiera incursionar en esta herramienta de participación comunitaria. Según dicha web el Design Thinking es un método para generar ideas innovadoras, que centra su eficacia en entender y dar solución a las necesidades reales de los usuarios. Esta noción proviene de la forma en la que trabajan los diseñadores de producto; de ahí su nombre, que en español se traduce de forma literal como "Pensamiento de diseño".

Nicholas y Oak (2020) exploran la tensión de las cualidades de "hacer o deshacer", de los detalles arquitectónicos en el aprendizaje y la realización del diseño, y además lo definen como el proceso material de unir elementos dispares y un lugar para relaciones sociales y profesionales complejas y para actividades de creación de significado. Y Lauff, Knight, Kotys-Schwartz y Rentschler (2020) mencionan que los prototipos son artefactos complejos y dinámicos que dan forma a las situaciones sociales durante el desarrollo del producto; rematan su estudio diciendo que los prototipos se codifican con información que los grupos y partes interesadas traducen, decodifican y vuelven a codificar.

\footnotetext{
2 Véase Design Thinking [http://www.designthinking.es/inicio/].
} 
También hay aspectos metodológicos a tener en cuenta a la hora de abordar las fenomenologías urbanas tales como los flujos peatonales, usos y subutilizaciones de espacios urbanos, entre otros, que son problemáticas o potenciales de intervenir. El orden espacial y las prácticas que realizan los habitantes de un lugar pueden modificarse ya que, si nos atenemos a lo planteado por Griffiths y Vaughan (2020):

Las descripciones cuantitativas de las redes de calles urbanas producidas por los análisis de sintaxis espacial se pueden utilizar para formular y probar hipótesis sobre patrones de movimiento urbano, encuentro y actividad socioeconómica en el pasado, que pueden ayudar en la interpretación de otros materiales de origen histórico para dar un panorama general y cuenta de la cultura espacial urbana (p. 488).

Uno de los aportes esenciales del Design Thinking es la importancia que adquiere el ciudadano/a y la comunidad en el diseño de sus proyectos de mejora espacial urbana, puesto que conoce los problemas de primera mano ya que los vive a diario. Se trata entonces de transmitir la forma en que piensan los diseñadores especializados al ciudadano/a del común. Tal vez en esto resida su importancia puesto que se trata de instruir en menesteres de diseño a una persona que no tiene ni la formación ni el conocimiento, sino que se la sensibiliza en busca de un cierto pensamiento crítico sobre su realidad local y se le conduce mediante cierto procedimiento a las formas de confrontar sus problemáticas para poder generar soluciones creativas que mitigue o solucione problemas reales de su vida cotidiana.

Otro aspecto preponderante es su proceso flexible de principios participantes entre los actores intervinientes, las relaciones de empatía, la definición del problema a solucionar, la ideación, los prototipos y el seguimiento que se le hace para revisar las aceptaciones y apropiaciones por parte de la comunidad objeto de intervención (Diagrama 2).

\section{Diagrama 2. Flujo clásico del Design Thinking}

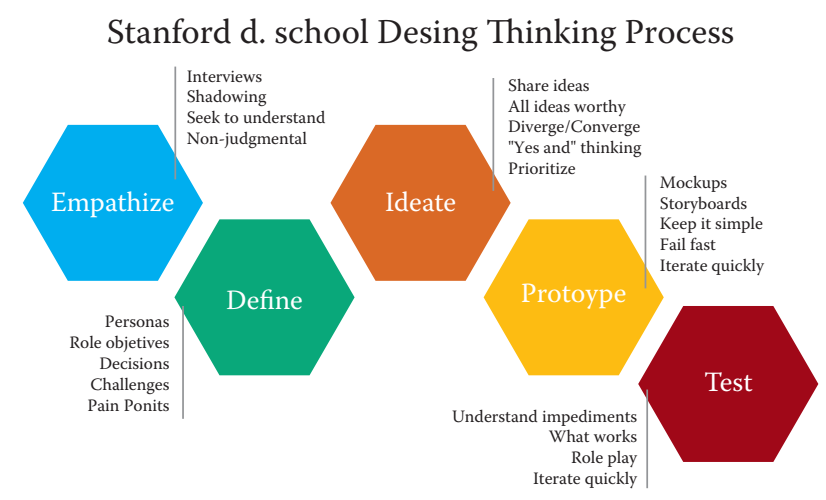

Fuente: Ferràs (2018).

\section{Hacking the city (Hackeando la ciudad)}

Este concepto es relativamente nuevo e implica una cierta noción de tecnocracia que se mantiene encerrada en los círculos artísticos y sólo se desvela cuando sus expresiones salen a la calle. Es poco conocido en los círculos elitistas de la ciencia puesto que son experimentaciones en el espacio público por lo general itinerantes, de ahí que sus apropiaciones ciudadanas algunas veces no sean entendidas. Esto se puede apreciar cuando se habla de los "hacks culturales" que no pueden anunciarse y se traduce en esa falta de comunicación con el usuario/a que lo va a observar, a vivir, a disfrutar y que puede originar incomprensión, descontextualización, incomodidad y posiblemente la no aceptación pues saca al urbanita de su cotidianidad (Sterling, 2011). Eso hace que sus resultados sean muy volubles, que queden en la ambigüedad y que, la mayoría de las veces, no supere el ámbito artístico y no cale en una posible iniciativa de apropiación ciudadana que lleve de verdad a repensar el paisaje urbano especialmente de lo marginal y lo excluido.

Cuando se habla de "Hackeo cultural" se hace de un tipo de manifestación que raya en lo subversivo y busca criticar, oponer resistencia, debilitar lo establecido en sus apariciones sin previo aviso en la calle. Esto lleva a pensar que los cambios de paradigma producen evoluciones que por lo 
general pueden generar impactos valederos para espacios urbanos que lo reclaman. Sin embargo para que ello ocurra hace falta mucha sensibilidad espacial, arquitectónica, urbana y sobre todo estética, para que su aparición sea aceptada y apropiada por la gente. Desde esa perspectiva puede ser una herramienta más en la búsqueda esperanzadora de autogestión espacial y la participación comunitaria, que conlleve a micro cambios culturales en algunos entornos urbanos de conflicto que pueden ser liderados por las nuevas generaciones.

Todas las intervenciones que se realizan tienen un impacto en la vida social urbana. El simple hecho de pintar una fachada y la calle, tal como ocurrió en la ciudad de Riga, Letonia (Figura 4), amplía visual y virtualmente la acera, ganándole espacio al vehículo para obsequiársela al peatón; propicia la interacción entre las personas viandantes y rompe con lo tradicional, con lo aceptado socialmente -esté mal o no-; busca un impacto inmediato para llamar la atención sobre la forma de pensar, la manera de actuar del ciudadano común y corriente, y propicia la generación de proyectos más sostenibles -y ojalá definitivos-, avalados por la ciudadanía cuando evidencia la mejora en la calidad de vida de esos micro espacios urbanos. En ese sentido, tal como afirman Tamara, Boyco y Letelirer (2018), se pretende

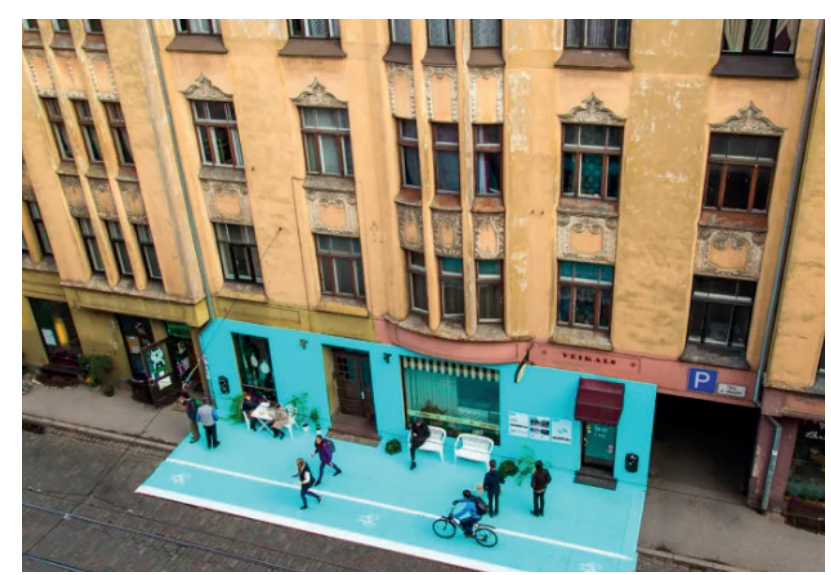

Figura 4. El impacto del color en la fachada y la acera cambia la cotidianidad urbana. Fuente: González (2016).
(...) fortalecer el poder de incidencia, negociación y acción colectiva de la ciudadanía, articulando a los y las vecinas, organizaciones y grupos que funcionan en escalas micro vecinales, de forma tal que emerja un nuevo actor, que visibilice las problemáticas socio urbanas en esta escala mayor y formule y gestione agendas de desarrollo ante las instituciones públicas y los actores privados (p. 10).

\section{Las intervenciones tácticas en la periferia marginal}

El urbanismo táctico es un concepto nuevo y vanguardista que invita a participar a ciertos actores de la ciudad, entusiastas activos de iniciativas de desarrollo local, pues maneja una escala espacial más humanizada -pequeña, si se quiere ser literal- y esto hace que su legitimidad en el territorio se convierta en una de sus premisas (Figura 5). Estas diferencias de escala y espacio implican que las intervenciones físico-espaciales tengan más flexibilidad en cuanto a metodologías, herramientas y formas de actuar. Estas intervenciones se pueden llevar a cabo con principios de diseño participativo o no y los agentes intervinientes pueden constituir un abanico muy amplio. Entonces los gobiernos locales, la academia, las organizaciones no gubernamentales, las comuni-

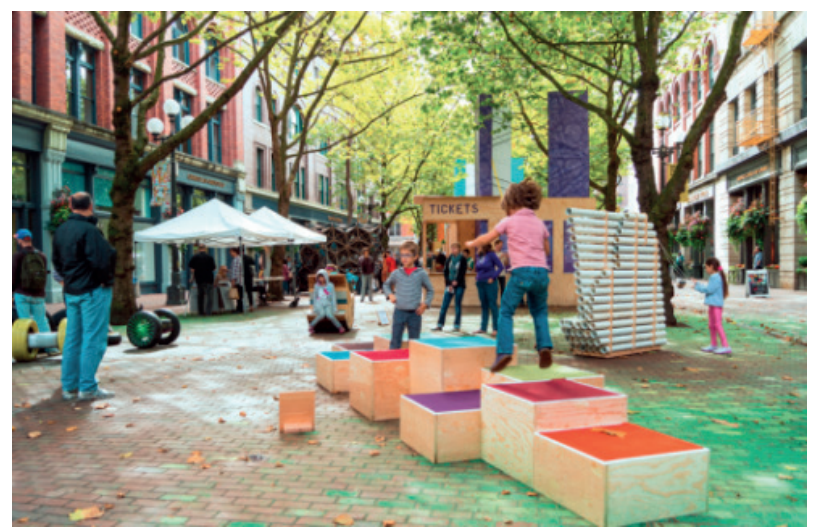

Figura 5. Urbanismo Táctico y transformación de la cotidianidad en un espacio público urbano. Fuente: Herrera Jaramillo (2020). 
dades organizadas con conocimiento de referentes estéticos o acompañamiento técnico, pueden contribuir a la resolución de problemáticas locales urbanas con proyectos piloto que busquen el mejoramiento de la calidad de vida.

Lo dicho anteriormente implica que son proyectos itinerantes de corto tiempo, con materiales de bajo costo lo que no garantiza una permanencia, de ahí su maleabilidad. Por sus características de implementación se puede sugerir algunos elementos a tener en cuenta: se debe iniciar con cierta sensibilidad espacial de las fenomenologías y cotidianidades locales que evidencien alguna problemática o aspectos "negativos" que no favorezcan la convivencia armónica; que representen peligro, ausencia de estética, deshumanización y falta de inclusión socio espacial; que tengan déficit y falta de calidad del espacio público, problemas ambiental locales, contaminación visual, auditiva, entre otros. Todo en su conjunto se debe convertir en una búsqueda de producción de lugares para el encuentro, la interacción social, la especulación de prácticas (Cedeño-Pérez, 2006), la recreación, el descanso, la contemplación y que obedezca a un cambio de la comunidad local y represente una porción de la ciudad que tiene la potencialidad de ser mejorado.

Después de la observación de la fenomenología negativa local, in situ se interpretan las cotidianidades para que la intervención a desarrollar responda a lógicas de uso de fácil apropiación por parte de la comunidad. Desde esa perspectiva cabe aclarar que el espacio intervenido aparentemente cambia su uso cotidiano, promueve una reflexión por parte del transeúnte, indaga sobre su corresponsabilidad de uso o no; por ello la propuesta debe tener premisas relacionadas con lo participativo, lo estético, lo ambiental, el confort y que sea de fácil entendimiento para la apropiación comunitaria y facilitadora de la cohesión social en su área de influencia, para que tenga el potencial de convertirse en referencia urbana.
La intervención debe hacerse con materiales de bajo costo, que implique un desarrollo fácil y rápido; puede tener principios de reciclaje o reutilización de materiales, modificación de objetos existentes, etc. En ese sentido se busca contrastar con el entorno para volverlo visible y de fácil acceso, entonces se hace necesario realizar un seguimiento en un tiempo prudencial que puede variar y que depende de la cobertura de su impacto si es a nivel local, barrial o sectorial, pues a mayor cobertura mayor tiempo de permanencia de la instalación o proyecto piloto.

El urbanismo estratégico tiene una filosofía relacionada con el pensar y actuar ágilmente, deja de lado los procesos largos y dispendiosos de la planificación urbana y busca un cambio de la cotidianidad local. Las intervenciones hechas en los entornos urbanos indagan sobre la aceptación o no de la ciudadanía y de esto depende la modificación de la propuesta inicial o la intervención posterior definitiva, de mayor costo y con materiales que aseguren su sostenibilidad en el tiempo y en últimas redunde en el mejoramiento de la calidad de vida.

Las relaciones entre el urbanismo táctico y la participación ciudadana pueden tener diferentes grados de conflictividad y en la mayoría de los casos depende de la propuesta de intervención con el proyecto piloto, su publicidad, la socialización hecha a los vecinos y vecinas, el mensaje que transmite, la instalación físico-espacial, el beneficio que presta, la comprensión y apropiación por parte de la población y si ese cambio de uso logra romper el paradigma de la cotidianidad, con una transformación del paisaje urbano que beneficia la existencia de los/as transeúntes; de aquellos que configuran la vida urbana, lo urbano, tal como lo enuncia Delgado (2018). En este caso vale la pena presentar el ejemplo de una calle de Medellín (Figura 6) en donde una intervención de urbanismo táctico, no sólo reduce la siniestralidad vial sino que también protege a las personas viandantes y ofrece un cambio estético del paisaje urbano (Secretaría de Movilidad de Medellín, 2017). 


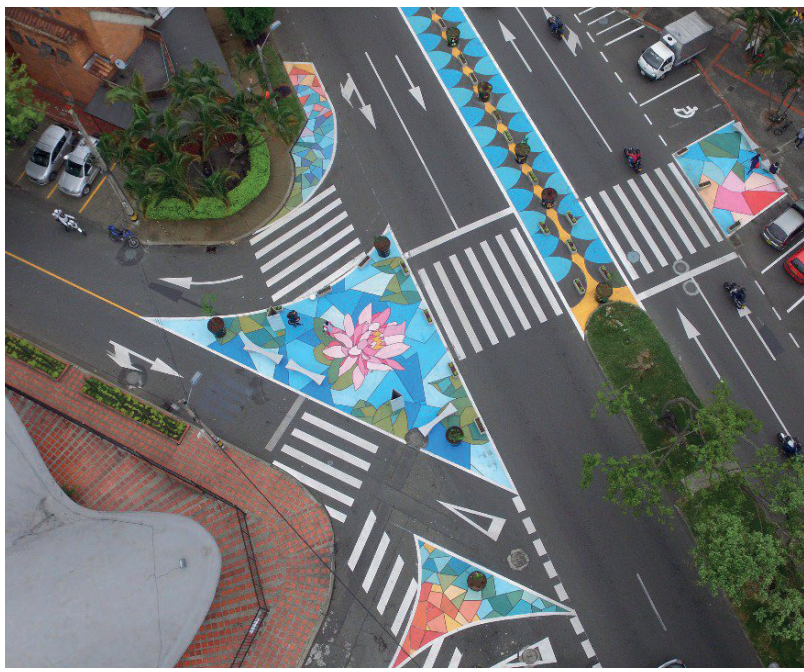

Figura 6. Ejemplo de urbanismo táctico en una calle de Medellín. Fuente: Secretaría de Movilidad de Medellín (2017).

Lo dicho hasta aquí también invita a preguntarse sobre las razones para utilizar el urbanismo táctico en una periferia marginal. Es importante tener en cuenta que la mayoría de referentes de proyectos a nivel mundial y local se han hecho en centralidades urbanas como, por ejemplo, en Times Square, Nueva York; en donde se llevó a cabo un proceso de peatonalización (Herrera Jaramillo, 2015), como ya se mencionó en un apartado anterior. Algunas de las referencias sobre urbanismo táctico, ya señaladas, evidencian un centralismo asiduo de intervenciones que por sus ubicaciones estratégicas han logrado un impacto global inmediato -ayudado por Internet- y ello entraña que las intervenciones en las periferias urbanas sean muy escasas. Para referenciar algunas se puede mencionar el caso de la ciudad de Pachuca, Hidalgo, México, en donde fueron intervenidas desde lo pictórico - murales individuales por casa pero que en su conjunto conforma un collage artístico-, 209 casas del barrio marginal Palmitas (Figura 7); un cambio del paisaje urbano de un barrio que muestra sus culatas hacia la ciudad y que sirve de lienzo para una obra de "arte urbano periférico" que se hizo con la participación de un colectivo de artistas junto con la comunidad (AFP, 2015).

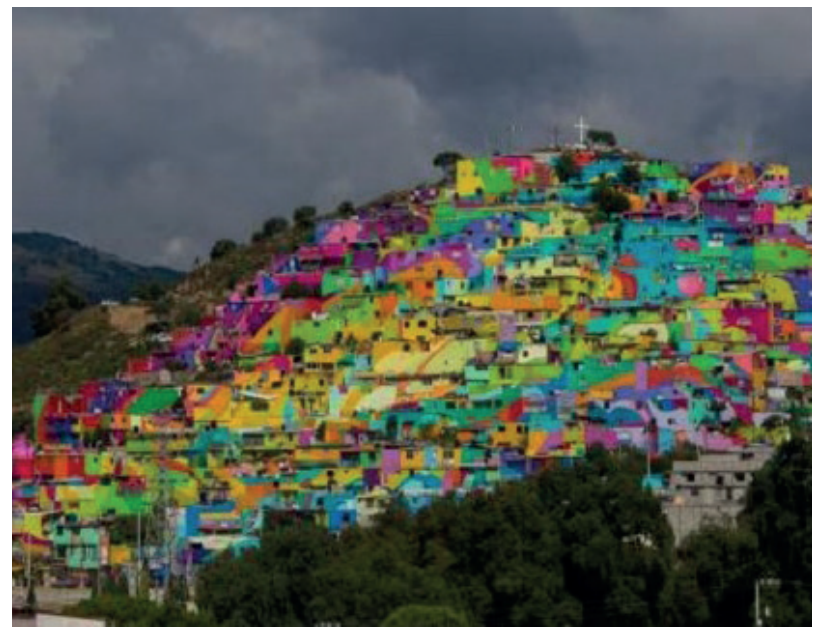

Figura 7. Intervención del barrio Palmitas, en Pachuca (México). Fuente: NicaLeaks (2016).

Sobre este tipo de intervenciones tácticas vale la pena tener en cuenta que su injerencia se centra en lo "cromático-social" más que en lo "esencial espacial marginal" y aunque es un aporte al paisaje urbano para observarse desde la distancia, necesita repintarse cada cierto tiempo; lo que implica una reflexión desde el punto de vista de su sostenibilidad y desde las estéticas populares que lo intervendrán, lo cambiarán, lo deteriorarán y lo apartarán de su iniciativa estética inicial. Esto significa, entre otras cosas, que su intemporalidad es dudosa y que, de todas maneras, no se puede ser inflexible en estos trabajos colaborativos con la comunidad porque son siempre un activo social de desarrollo urbano local. Más allá se puede percibir que, aunque no se solucionan todos los problemas ambientales de los asentamientos humanos, ni sus infraestructuras y menos los aspectos socioeconómicos, estas intervenciones en las periferias urbanas podrían crear identidad barrial, apropiación de sus entornos, convivencia pacífica y espacios de encuentro dignos. En ese sentido se puede descubrir en sus habitantes líderes, vocaciones, emprendimientos, apropiaciones y aproximaciones de inclusión para contrarrestar algunas de las fenomenologías originadas por las segregaciones socio espaciales que son una constante en la ciudad latinoamericana. 


\section{Conclusiones}

En la periferia marginal pueden existir lugares subutilizados con potencial de constituirse en comarcas de encuentro, de lúdica, de recreación, de esparcimiento y/o intersticios urbanos como zonas verdes, taludes, culatas o áreas abandonadas que pueden ser de uso comunitario. Estos son los vacíos que desde lo urbano arquitectónico y con metodologías del urbanismo táctico cobran real importancia y constituyen una oportunidad en la búsqueda de soluciones a algunas problemáticas urbanas de sectores marginales. $\mathrm{Y}$ en ese sentido manifestaciones de vanguardia como Design thinking, Hacking the City, urbanismo táctico, diseño y planeación participativa, constituyen un abanico amplio de formas de intervención en los territorios periféricos.

La intervención táctica en periferias marginales a partir de la participación ciudadana, es un tema que todavía parece novedoso en el sentido de que si se revisan diferentes referentes estéticos globales y/o locales, se vislumbra que la mayoría de estos ejemplos se limita a injerencias pictóricas en barrios marginados o a trabajos realizados en centralidades urbanas que siempre han dejado de lado los barrios vulnerables.

Se pueden presentar limitaciones en el desarrollo de proyectos piloto en territorios periféricos tradicionalmente excluidos ya que a veces no son lo suficientemente entendidos debido, quizá, a que la vida cotidiana de la comunidad está signada por la exclusión socio-espacial, el desempleo, la violencia, los problemas socioeconómicos y la vulnerabilidad. Y estas intervenciones la mayoría de las veces se hacen sobre el espacio público o zonas verdes subutilizadas lo cual puede ser visto como algo accesorio y superfluo; un lujo que no es importante ni prioritario para la resolución de las necesidades básicas de la comunidad. Por ello merece la pena reflexionar sobre el urbanismo táctico y la participación ciudadana en las periferias marginales; verificar si hay una relación fuerte entre sí o tal vez su complejidad conceptual es frágil en territorios donde existen otras prioridades vitales cotidianas como la vivienda, la comida, la salud, el empleo, los servicios públicos pero que constituye una forma de romper con esas realidades diarias porque procura la dignidad humana por encima de lo físico-espacial.

Por último, es necesario que la academia se convierta en el primer espacio de reflexión, de creación y transferencia de conocimiento y que proponga diferentes actuaciones de impacto en las comunidades marginales para contribuir al mejoramiento de sus entornos y por ende a su calidad de vida.

\section{Referencias bibliográficas}

AFP (2015). El arte cambia la vida de un barrio mexicano de escasos recursos. Diario El comercio. Recuperado de: [https:// www.elcomercio.com/tendencias/artepalmitas-mexico-criminalidad-murales.html].

Brenna, M., Bucci, V., Falvo, M. C., Foiadelli, F., Ruvio, A., Sulligoi, G. y Vicenzutti, A. (2020). A review on energy efficiency in three transportation sectors: Railways, electrical vehicles and marine. Energies, 13(9). DOI: [https://doi. org/10.3390/en13092378].

Cedeño-Pérez, M.C. (2006). Relaciones sociales y prácticas de apropiación espacial en los parques públicos urbanos. (El caso del Parc de Les Planes de L'Hospitalet de Llobregat - Barcelona) (Tesis de doctorado). Universidad de Barcelona, Barcelona. Recuperado de: [http://diposit.ub.edu/ dspace/handle/2445/35202].

Christof, K. (2017). The citizen professional, mediatization, and the creation of a public domain. Culture Unbound, 9(3), pp. 279-306. DOI: [https://doi.org/10.3384/cu.2000.1525. 1793279].

Ciocoletto, A (2014). Espacios para la vida cotidiana, Auditoria de calidad urbana con perspectiva de género (Tesis doctoral). Universitat Politècnica 
de Catalunya. Departament de Construccions Arquitectòniques. Barcelona. Recuperado de: [https://tdx.cat/handle/10803/275979\#page=1].

Cubero, M. E. P. (2019). Citizen participation in socio-environmental movements in Latin America (La participación ciudadana de los movimientos socioambientales en América Latina). Revista Colombiana de Sociología, 42(1), pp. 135-156. DOI: [https://doi.org/10.15446/ rcs.v42n1.73023].

Dalla-Torre, A. (2017). Territorial governance and the Territorial Planning Plans: The case of the province of Mendoza, Argentina [Governança territorial e os planos do ordenamento do território: o caso da província de mendoza, Argentina]. Bitácora Urbano Territorial, 27(1), pp. 47-54. DOI: [https://doi.org/10.15446/bitacora.v27n1.47597].

Delgado, M. (2018). El urbanismo contra lo urbano. La ciudad y la vida urbana en Henri Lefebvre. REVISTARQUIS, 7(1), pp. 65-71. Recuperado de: [http://diposit.ub.edu/dspace/ bitstream/2445/121605/1/676055.pdf].

Design Thinking (2020). ¿Qué es el Design Thinking? Recuperado de: [https://www.designthinking.es/inicio/].

Diz, C. (2018). Tactics of the body: Activism and resistance in the crisis city (Tácticas del cuerpo: Activismo y resistencia en la ciudad en crisis). Revista de Dialectologia y Tradiciones Populares, 73(1), pp. 127-152. DOI: [https://doi. org/10.3989/rdtp.2018.01.005].

Ferràs, X. (2018). Inverse design thinking. Recuperado de: [https://xavierferras.com/2018/10/ inverse-design-thinking-2/].

Gehl, J. (2014). Ciudades para la gente. Buenos Aires: Infinito.

Gené, G. y Ferré, J. (2014). Peatonalización de Nueva York. Paisea: revista de paisajismo, 29, pp. 80-86. Recuperado de: [http://www.paisea. com/wp-content/uploads/PAISEA-29-ART-2a. pdf].
Griffiths, S. y Vaughan, L. (2020). Mapping spatial cultures: Contributions of space syntax to research in the urban history of the nineteenth-century city. Urban History, 47(3), pp. 488-511. DOI: [https://doi.org/10.1017/ S0963926820000206].

González, E. (17 de mayo de 2016). Finalistas del Premio Europeo de Espacio Público. Recuperado de: [https://www. edgargonzalez.com/2016/05/17/finalistas-delpremio-europeo-de-espcio-pblico/].

Herrera Jaramillo, M. (6 de abril de 2015). tác-tico [Entrada en blog]. Recuperado de: [http:// ciudaduideloja.blogspot.com/2015/04/autoraarq.html].

Herrera Jaramillo, M. (4 de mayo de 2020). Urbanismo táctivo. [Entrada en blog]. Recuperado de: [https://mayradelcisne.wordpress.com/tag/ urbanismo-tactico/].

Lauff, C. A., Knight, D., Kotys-Schwartz, D. y Rentschler, M. E. (2020). The role of prototypes in communication between stakeholders. Design Studies, 66, pp. 1-34. DOI: [https://doi. org/10.1016/j.destud.2019.11.007].

NicaLeaks (6 de junio de 2016). Los muralistas mexicanos pintaron un barrio entero. Recuperado de: [http://nicaleaks.com/2016/06/06/ los-muralistas-mexicanos-pintaron-un-barrioentero/].

Nicholas, C. y Oak, A. (2020). Make and break details: The architecture of design-build education. Design Studies, 66, pp. 35-53. DOI: [https:// doi.org/10.1016/j.destud.2019.12.003].

Noriega, A., Aburto, F. y Montecinos, E. (2016). Presupuestos participativos en Chile y su contribución a la inclusión social. Iconos. Revista de Ciencias Sociales (56), pp. 203-218. Recuperado de: [https://www.redalyc.org/articulo. oa?id=509/50947321011].

Pindado, F. (2004). Gobierno local y participación ciudadana. Intervención Psicosocial, 13 (3), pp. 
307-323. Recuperado de: [https://www.redalyc. org/articulo.oa?id=1798/179817830002].

Plataforma Urbana (2013). "Pittaki" Vecinos de Atenas donan sus lámparas para iluminar las calles. Recuperado de: [https://www. plataformaurbana.cl/archive/2013/02/13/\%E2\% 80\%9Cpittaki\%E2\%80\%9D-vecinos-de-atenasdonan-sus-lamparas-para-iluminar-las-calles/] .

Ramos-Vidal, I. (2017). Community dynamics among displaced and non-displaced residents in socially excluded areas of Barranquilla, Colombia (Dinâmicas comunitárias em deslocados e não deslocados residentes em áreas de exclusão social em Barranquilla, Colômbia). Revista de Estudios Sociales, 2017(60), pp. 49-61. DOI: [https://doi.org/10.7440/res60.2017.04].

Samper, J., Ortiz, C. y Soto, J. (2014). Repensando la informalidad, estrategias de co-producción del espacio urbano, Medellín, Comuna 8. Medellín: Universidad Nacional de Colombia.

Secretaría de Movilidad de Medellín (23 de octubre de 2017). Con el fin de reducir la siniestralidad y proteger al peatón implementaremos el urbanismo táctico [Tweet]. Recuperado de: [https://twitter.com/sttmed/ status/922476206301351936/photo/1].
Signorelli, G. V. (2016). The role of popular territories in participatory budgeting of the cities of Porto Alegre and Rosario (El rol de los territorios populares en el presupuesto participativo de las ciudades de porto alegre y rosario). America Latina Hoy, 72, pp. 103-128. DOI: [https://doi. org/10.14201/alh201672103128].

Stang, J. I. (2014). La Nueva York peatonal. La recuperación de la experiencia en el espacio público. Bifurcaciones: Revista de estudios culturales urbanos, 18,pp.1-2.Recuperadode:[http://www. bifurcaciones.cl/bifurcaciones/wp-content/ uploads/2014/11/bifurcaciones_018_Stang. pdf].

Sterling, B. (14 de junio de 2011). Hacking the City [Entrada en blog]. Wired. Recuperado de: [https://www.wired.com/2011/06/hackingthe-city/].

Tamara, J., Boyco, P. y Letelirer, F. (2018). Manual metodológico acción territorial vecinal. Modelo de innovación social para el desarrollo participativo de territorios vecinales. Maule: Universidad Católica del Maule.

Ziccardi, A. (2004). Participación ciudadana y políticas sociales del ámbito local. México: Universidad Autónoma de México. 